\title{
The role of multicomponent therapy in the metabolic syndrome, inflammation and cardiovascular risk in obese adolescents
}

\author{
Deborah C. L. Masquio $^{1 *}$, Aline de Piano ${ }^{1}$, Raquel M. S. Campos ${ }^{1}$, Priscila L. Sanches ${ }^{1}$, June Carnier $^{1}$, \\ Flávia C. Corgosinho ${ }^{1}$, Bárbara D. M. Netto ${ }^{1}$, Joana P. Carvalho-Ferreira ${ }^{2}$, Lila M. Oyama ${ }^{1,3}$, \\ Claudia M. O. Nascimento ${ }^{1,3}$, Marco T. de Mello ${ }^{1}$, Sergio Tufik ${ }^{4}$ and Ana R. Dâmaso ${ }^{1,2 *}$ \\ ${ }^{1}$ Post-Graduate Program of Nutrition, Universidade Federal de São Paulo, São Paulo-SP, Brasil \\ ${ }^{2}$ Post-Graduate Program of Interdisciplinary Health Science, Universidade Federal de São Paulo, Santos-SP, Brasil \\ ${ }^{3}$ Department of Physiology, Universidade Federal de São Paulo, São Paulo-SP, Brasil \\ ${ }^{4}$ Department of Psycobiology, Universidade Federal de São Paulo, São Paulo-SP, Brasil
}

(Submitted 9 October 2014 - Final revision received 20 February 2015 - Accepted 11 March 2015 - First published online 24 April 2015)

\begin{abstract}
Obesity is characterised by low-grade inflammation, which increases the metabolic syndrome (MetS) and cardiovascular risks. The aim of the present study was to verify the role of multicomponent therapy in controlling the MetS, inflammation and carotid intima-media thickness (cIMT) in obese adolescents. The second aim was to investigate the relationships between adipokines, the MetS parameters and cIMT. A total of sixty-nine obese adolescents participated in the present study and completed 1 year of multicomponent therapy (a combination of strategies involving nutrition, psychology, physical exercise and clinical therapy), and were divided according to their MetS diagnosis as follows: MetS ( $n$ 19); non-MetS ( $n$ 50). Blood analyses of glucose, lipid and adipokine concentrations (adiponectin, leptin, plasminogen activator inhibitor 1 (PAI-1) and C-reactive protein) were collected. Insulin resistance was assessed using the homeostasis model assessment for insulin resistance, quantitative insulin sensitivity check index and homeostasis model assessment-adiponectin. cIMT and visceral and subcutaneous fat were estimated using ultrasonography. At baseline, the MetS group presented higher waist circumference, glucose and insulin levels, and systolic and median blood pressures compared with the non-MetS group. After therapy, both groups showed improvements in the anthropometric profile, body composition, insulin level, insulin resistance, insulin sensibility, TAG and VLDL-cholesterol, adiponectin, leptin and PAI-1 levels, blood pressure and cIMT. The prevalence of the MetS was reduced from 27.5 to $13.0 \%$. Metabolic syndrome patients showed resistance in the attenuation of total cholesterol and LDL-cholesterol (LDL-C) levels and leptin:adiponectin and adiponectin:leptin ratios. In the MetS group, the variation in the adiponectin:leptin ratio was correlated with variations in glucose, insulin sensibility, total cholesterol, LDL-c and systolic blood pressure. Additionally, the number of MetS parameters was correlated with the carotid measurement. Moreover, the variation in cIMT was correlated with the variations in insulin sensibility, total cholesterol and LDL-c. For the entire group, the number of MetS alterations was correlated with the leptin level and leptin:adiponectin ratio and adiponectin:leptin ratio after therapy. In conclusion, multicomponent therapy was effective in controlling the MetS, inflammation and cIMT in the obese adolescents. However, the MetS patients showed resistance in the attenuation of the atherogenic lipid profile and leptin:adiponectin ratio and adiponectin:leptin ratio. These results suggest that the MetS patients have increased cardiovascular risks, and that it is important to attempt to control the inflammatory process that occurs due to obesity in clinical practice in order to improve the health of adolescents.
\end{abstract}

Key words: Obesity: Metabolic syndrome: Inflammation: Carotid intima-media thickness: Leptin: Adiponectin

The rise in childhood obesity is one of the most alarming public health issues, with approximately 110 million children diagnosed with obesity worldwide ${ }^{(1)}$. Moreover, childhood obesity frequently persists into adulthood, and it has been reported that up to $80 \%$ of obese children become obese adults. Excessive fat adipose tissue has been associated with

Abbreviations: cIMT, carotid intima-media thickness; DBP, diastolic blood pressure; GEO, Interdisciplinary Obesity Program/Obesity Study Group; HDL-C, HDL-cholesterol; HOMA-AD, homeostasis model assessment-adiponectin; HOMA-IR, homeostasis model assessment for insulin resistance; hs-CRP, highsensitivity C-reactive protein; LDL-C, LDL-cholesterol; MBP, mean blood pressure; MetS, metabolic syndrome; PAI-1, plasminogen activator inhibitor 1; QUICKI, quantitative insulin sensitivity check index; SBP, systolic blood pressure; VLDL-C, VLDL-cholesterol.

*Corresponding authors: D. C. L. Masquio, fax +55 11 30888995, email deborahmasquio@yahoo.com.br; A. R. Dâmaso, fax +55 11 30888995, email ana.damaso@unifesp.br 
chronic systemic low-grade inflammation, which is related to metabolic complications, such as the metabolic syndrome $(\mathrm{MetS})^{(1,2)}$

The prevalence of the MetS, which is characterised by a combination of cardiometabolic alterations including elevated TAG, reduced HDL, high blood pressure, impaired fasting glucose and increased abdominal circumference, has also increased ${ }^{(3)}$. In a sample of fifty-seven obese Brazilian adolescents, it has been demonstrated that $33 \%$ had a diagnosis of the MetS ${ }^{(4)}$. In addition, the MetS has been associated with incident coronary artery disease, stroke and cardiovascular events in adults ${ }^{(3,5)}$.

Poor nutritional habits and sedentarism are collectively related to increased obesity during adolescence ${ }^{(6)}$, which reinforces the importance of intervention studies that have aimed to increase dietary quality and physical activity levels during this phase of life ${ }^{(7)}$. Multicomponent therapy plays an important role in improving outcomes and controlling metabolic alterations in obese adolescents, because this model of intervention has the main objective of changing lifestyle factors and promoting health ${ }^{(8,9)}$. The combination of weight loss therapy with nutritional, psychological and physical exercise support has been shown to be effective, not only for obesity control but also for other related disorders, including nonalcoholic fatty liver disease, asthma and binge eating, in obese adolescents $^{(10)}$. However, further exploration is needed to identify mechanisms that can better control the MetS parameters in obese adolescents to reduce cardiovascular risk factors and to improve the quality of life and health early in life.

Human obesity is characterised by high levels of proinflammatory circulating adipokines, such as leptin, in a condition known as hyperleptinaemia, in addition to elevations in C-reactive protein (CRP), plasminogen activator inhibitor 1 (PAI-1) and a reduction in adiponectin, which is an important anti-inflammatory adipokine ${ }^{(4,11)}$. This imbalance in pro/anti-inflammatory adipokines results in a chronic inflammatory state, which is thought to be associated with an increased incidence of the MetS in youth, leading to increases in cardiovascular risks ${ }^{(11,12)}$.

Leptin is an adipose tissue-derived hormone with functions related to human energy homeostasis and weight control under physiological conditions. However, hyperleptinaemia can be associated with disorders related to endothelial dysfunction $^{(13)}$. In fact, previous findings have demonstrated that a high concentration of leptin in obese adolescents is related to cardiovascular risks and the atherosclerotic process. Hyperleptinaemic obese adolescents are unable to increase adiponectin levels after weight loss therapy, which suggests a role of hyperleptinaemia in the impaired attenuation of inflammation, thereby leading to a decrease in vascular protection $^{(8)}$

Moreover, low levels of adiponectin are related to obesity. Evidence has indicated that hypoadiponectinaemia is associated with increased cardiovascular risks, such as the MetS, type 2 diabetes, atherosclerosis and CVD, in obese patients ${ }^{(14,15)}$.

Given that obesity and the MetS are linked to low-grade inflammation, and that they both have the potential to influence the severity of CVD, strategies for controlling these conditions may improve metabolic status in obese patients. Thus, the first aim of the present study was to verify the role of multicomponent therapy in controlling the MetS, inflammation and carotid intima-media thickness (cIMT) in obese adolescents. The second objective was to investigate the possible relationships between pro/anti-inflammatory adipokines, the MetS parameters and cIMT in the analysed population.

\section{Methods}

\section{Subjects}

A total of 132 obese adolescents were recruited to participate in the Interdisciplinary Obesity Program/Obesity Study Group (GEO) of the Universidade Federal de São Paulo/Escola Paulista de Medicina. At the beginning of the year, the project was published in the media, newspapers and magazines in São Paulo to recruit adolescents. The inclusion criteria were age (15-19 years), post-pubertal Tanner stage $\geq 5^{(16)}$ and BMI $>95$ th percentile of CDC guidelines ${ }^{(17)}$. The exclusion criteria included an identified genetic disease, previous drug use, chronic alcohol consumption, pregnancy and the inability to perform physical activities based on an electrocardiogram test. The sample size for the present study was a convenience sample determined by the number of participants meeting the GEO inclusion criteria.

For the present analysis, we excluded those patients who did not complete the therapy by attending more than $75 \%$ of the treatment sessions and evaluations for 1 year. Thus, sixty-nine obese adolescents (forty-two girls and twenty-seven boys) were evaluated in the present study and concluded weight loss therapy in 1 year. The patients were distributed into two groups as follows: diagnosed with the MetS and not diagnosed with the MetS.

A MetS diagnosis was made when the waist circumference was higher than the 90th percentile for age and sex in association with two or more International Diabetes Federation (IDF) criteria, as follows: $\mathrm{HDL} \leq 1.3 \mathrm{mmol} / \mathrm{l} \quad(\leq 50 \mathrm{mg} / \mathrm{dl})$ (girls) and $\leq 1.0 \mathrm{mmol} / \mathrm{l} \quad(\leq 40 \mathrm{mg} / \mathrm{dl})$ (boys); $\mathrm{TAG}>1.7$ $\mathrm{mmol} / \mathrm{l}(>150 \mathrm{mg} / \mathrm{dl}) ;$ glucose $>5.6 \mathrm{mmol} / \mathrm{l} \quad(>100 \mathrm{mg} / \mathrm{dl})$; blood pressure $\geq 130 / 85 \mathrm{mmHg}^{(18)}$.

The present study was conducted according to the principles of the Declaration of Helsinki, was approved by the Institutional Ethical Committee (no. 72 538) and was registered with ClinicalTrials.gov (no. NCT01358773).

\section{Medical screening}

An endocrinologist completed a clinical interview to determine the inclusion and exclusion criteria. All subjects underwent electrocardiograms. Thereafter, the volunteers started the multicomponent weight loss intervention. All measurements were performed at baseline and after 1 year.

\section{Anthropometric measurements and body composition}

Body mass $(\mathrm{kg})$, height $(\mathrm{m})$, BMI $\left(\mathrm{kg} / \mathrm{m}^{2}\right)$ and waist circumference were measured using standard procedures ${ }^{(19)}$. 
Body composition was estimated by plethysmography air displacement in a BOD POD system (version 1.69; Life Measurement Instruments) ${ }^{(20)}$

Visceral and subcutaneous fat were estimated using abdominal ultrasonography by the same physician blinded to the subject assignment groups. A $3.5 \mathrm{MHz}$ multifrequency transducer (broad band) was used to reduce the risk of misclassification. Subcutaneous fat was defined as the distance between the skin and the superficial plane of the rectus abdominal muscle. Visceral fat was defined as the distance between the deep plane of the same muscle and the anterior wall of the $\operatorname{aorta}^{(21)}$

\section{Carotid intima-media thickness measurements}

CIMT was measured using high-resolution ultrasound equipment (Logic 5 and Logic 7; General Electric) with a $7-14 \mathrm{MHz}$ linear array transducer. The same experienced radiologist performed all scans, and was unaware of the medical and biochemical statuses of the subjects; three repeated measurements of the right and left common carotid deep wall were obtained at $2 \mathrm{~cm}$ proximal to the bulb bifurcation. The distance between the leading edge of the first bright line on the deep wall (lumenintima interface) and the leading edge of the second bright line (media-adventitia interface) indicated the cIMT of the deep wall. The mean of the higher measurements for each side was used as the CIMT $^{(22)}$.

\section{Serum analysis}

Blood samples were collected after a $12 \mathrm{~h}$ overnight fast. The serum was separated, and concentrations of glucose, insulin, total cholesterol, HDL-cholesterol (HDL-C), LDL-cholesterol (LDL-C), VLDL-cholesterol (VLDL-C) and TAG were determined by enzymatic colorimetric methods (CELM) immediately after blood collection. The serum samples were stored at $-80^{\circ} \mathrm{C}$ for subsequent adipokine analyses. Leptin, adiponectin, PAI-1 and high-sensitivity CRP (hs-CRP) were measured with an ELISA kit from R\&D Systems. The CV for the adipokine procedure were as follows: adiponectin (4.5\%); leptin ( $4.07 \%)$; PAI-1 (3.89\%); hs-CRP (7.90\%). For the present study, the leptin data were analysed according to the reference values described by Gutin et al. ${ }^{(23)}$. The pro-inflammatory leptin:adiponectin ratio and anti-inflammatory adiponectin: leptin ratio were calculated.

Insulin resistance was determined using the homeostasis model assessment for insulin resistance (HOMA-IR) according to the following equation ${ }^{(24)}$ :

$$
\begin{aligned}
\text { HOMA-IR }= & \text { fasting insulin }(\mu \mathrm{U} / \mathrm{ml}) \\
& \times \text { fasting blood glucose }(\mathrm{mmol} / \mathrm{l}) / 22.5
\end{aligned}
$$

The homeostasis model assessment-adiponectin (HOMAAD) was calculated as follows ${ }^{(25)}$ :

$$
\begin{aligned}
\text { HOMA-AD }= & \text { fasting glucose }(\mathrm{mg} / \mathrm{dl}) \\
& \times \text { fasting insulin }(\mu \mathrm{U} / \mathrm{l}) / \operatorname{adiponectin}(\mu \mathrm{g} / \mathrm{ml}) .
\end{aligned}
$$

Insulin sensitivity was determined by the quantitative insulin sensitivity check index (QUICKI) as follows ${ }^{(26)}$ :

$$
\begin{aligned}
\text { QUICKI }= & 1 /(\log \text { fasting insulin }(\mu \mathrm{U} / \mathrm{ml}) \\
& +\log \text { fasting glucose }(\mathrm{mg} / \mathrm{dl})) .
\end{aligned}
$$

The HOMA-IR cut-off value adopted for adolescents was $3 \cdot 16^{(27)}$ and that of insulin was $15 \cdot 0^{(28)}$. (To convert insulin in $\mu \mathrm{U} / \mathrm{ml}$ to $\mathrm{pmol} / \mathrm{l}$, multiply by $6 \cdot 945$; to convert glucose in $\mathrm{mg} / \mathrm{dl}$ to $\mathrm{mmol} / \mathrm{l}$, multiply by $0 \cdot 0555$.)

\section{Blood pressure}

Systolic blood pressure (SBP) and diastolic blood pressure (DBP) were measured on the right arm using a mercurygravity manometer (Unitec) with the appropriate cuff size; two measurements were performed after the subjects had been sitting for at least $5 \mathrm{~min}$, and the mean value was used for the analyses. Mean blood pressure (MBP) was calculated as follows:

$$
\mathrm{MBP}=\mathrm{DBP}+((\mathrm{SBP}-\mathrm{DBP}) / 3) .
$$

\section{Research design}

The multicomponent obesity therapy consisted of clinical, nutritional and psychological therapies and exercise training for 1 year, as detailed below.

Clinical therapy. Each month, the obese adolescents visited the endocrinologist with their parents to address their health and clinical parameters. For each of these visits, the entire GEO team was also present. The doctor monitored and evaluated all of the adolescents' clinical examinations and treated health problems during therapy. The medical follow-up included an initial medical history and a physical examination including blood pressure, cardiac frequency, and body weight, and the adolescents were assessed for their adherence to the multicomponent therapy. The team discussed some possible lifestyle changes to promote their health with the adolescents and their parents.

Nutritional therapy. Once a week, the adolescents had a nutritional orientation, and were provided information on the food pyramid, diet record assessment, weight loss, highfat diets, food labels, dietetics, fat-free and low-energy foods, fast food calories and nutritional composition, good nutritional choices on special occasions, healthy sandwiches, shakes and products to promote weight loss, functional foods, and decisions on food choices. The lessons were taught by trained nutritionists to encourage the adolescents to follow healthy eating habits according to the food pyramid ${ }^{(29)}$

Each month, all patients received an individual nutritional consultation. Dietary energy intake was set at the level recommended by the dietary reference intake for subjects with low levels of physical activity of the same age and sex following a balanced diet ${ }^{(30)}$. No pharmacotherapies or antioxidants were recommended. At the individual nutritional consultation, some adjustments were made in the diet plan to adjust food habits and health.

At the beginning and at the end of the study, a 3-d dietary record was collected. Because most obese people 
under-report their food consumption, each adolescent was asked to record diet with the help from his/her parents. These dietary data were transferred to a computer by the same dietitian, and energy intake and nutrient composition were analysed by a PC programme developed at the Universidade Federal de São Paulo (Nutwin software for Windows; version 1.5) that used data from Western and local food tables.

Exercise therapy. The adolescents participated in personalised training for $60 \mathrm{~min} / \mathrm{session}$, three times a week (180 min/week) under the supervision of a sports therapist. The exercise protocol was based on the American College of Sports Medicine (ACSM) recommendations ${ }^{(31)}$ adapted by Foschini et al. ${ }^{(32)}$ of combined exercise, including $30 \mathrm{~min}$ of aerobic training plus $30 \mathrm{~min}$ of resistance training per session.

The aerobic training consisted of running on a motor-driven treadmill (Model TR 9700HR; Life Fitness) at a cardiac frequency intensity representing the ventilatory threshold I (50-70\% of the maximum oxygen uptake), as determined by the results of an initial oxygen uptake test for aerobic exercise. The heart rate was controlled by physiologists, and was measured with a cardiometer (Polar-Model ${ }^{\circ}$ FS1) at intervals of $5 \mathrm{~min}$ during the training sessions. After 6 weeks, aerobic tests were performed to assess physical capacity to make adjustments in physical training intensity ${ }^{(33)}$.

Resistance training exercises targeted each of the main muscle groups. The sequence of physical exercises used large muscle groups before small ones, multiple-joint before single-joint exercises and higher-intensity before lowerintensity exercises. The exercises included the bench press, leg press, sit-ups, lat pull-downs, hamstring curls, lowerback exercises, the military press, calf raises, arm curls and triceps pushdowns. The first 2 weeks of the resistance training programme involved adaptation to training and learning the movements (three sets of a maximum of fifteen to twenty repetitions). Following this period, the training load was adjusted by inversely modifying the volume and intensity, decreasing the number of repetitions to between six and twenty for three sets. The intensity of resistance training was quantified as a percentage of the one-repetition maximum and was continued until volitional fatigue for each of the major muscle groups. These factors were adapted to each individual's habitual physical activity, physical function, health status, exercise responses and stated goals, as recommended for universal screening to enhance the safety of exercise. The rest interval between the series and exercises was as follows: fifteen to twenty repetition maximum $=45 \mathrm{~s}$; ten to twelve repetition maximum $=1 \mathrm{~min}$; six to eight repetitions maximum $=1.5 \mathrm{~min}^{(33)}$.

Psychological therapy. The adolescents had a weekly psychological support group session to help them address their emotions, the relationship between their feelings and food consumption, and to discuss body image and eating disorders (bulimia, anorexia nervosa and binge eating, and their signs, symptoms and health consequences). Diagnoses of common psychological problems associated with obesity, such as depression, anxiety, body image disturbances, binge eating and bulimic symptoms, were established by validated questionnaires ${ }^{(34-38)}$. Individual psychological therapy was recommended when individuals were identified with nutritional and behavioural problems.

\section{Statistical analyses}

The distributional assumptions were verified by the Kolmogorov-Smirnov test. Parametric variables were expressed as the means and standard deviations, whereas non-parametric variables were expressed as the median (minimummaximum). Comparisons between measurements at baseline and after the weight loss intervention were performed using dependent $t$ tests for parametric variables and the Wilcoxon signed-rank test for non-parametric variables. Comparisons between groups at baseline and at the end of the therapy were made using the independent $t$ test for parametric variables or Mann-Whitney test for non-parametric variables. The Pearson and Spearman correlations were performed to assess the direction and strength of the relationships between the study variables. Simple linear regression analysis was applied. The McNemar $\chi^{2}$ test was used to verify the changes in the prevalence at baseline and after therapy. All data were analysed using STATISTICA 7.0 (Statsoft), and the significance level was set at a $P<0.05$.

\section{Results}

The effect of multicomponent therapy for the entire group

A total of 132 obese adolescents were enrolled in the programme; however, sixty-nine obese adolescents completed 1 year of the multicomponent weight loss therapy and more than $75 \%$ of the treatment sessions. It is important to note that no differences were observed in any variables between the completers and non-completers. In the present study, the patients were analysed in two groups according to MetS diagnosis, as follows: MetS ( $n$ 19) and non-MetS ( $n$ 50). The results are presented for the entire study population, and there were no significant sex differences in the prevalence of the MetS or its variables.

The prevalence of the MetS was reduced from $27 \cdot 5$ to $13.0 \%$ after 1 year of multicomponent therapy. We also observed significant reductions in the prevalence of the following altered MetS parameters after 1 year: waist circumference; SBP; DBP; hyperinsulinaemia; hyperleptinaemia (Fig. 1(a)). An increase was observed in the prevalence of patients with 0 and 1 alterations. However, there was a reduction in the prevalence of those with 2, 3 and 4 alterations (Fig. 1(b)).

\section{The effect of multicomponent therapy on the non-metabolic syndrome group}

After therapy, the non-MetS group presented significant reductions in body mass, BMI, body fat, waist circumference, visceral and subcutaneous fat and the visceral:subcutaneous fat ratio (Table 1). Significant improvements in insulin, HOMA-IR, QUICKI, total cholesterol, LDL-C, VLDL-C, TAG, SBP, DBP, MBP and energy intake were also observed. Fat-free mass (kg and \%) significantly increased. With regard to 


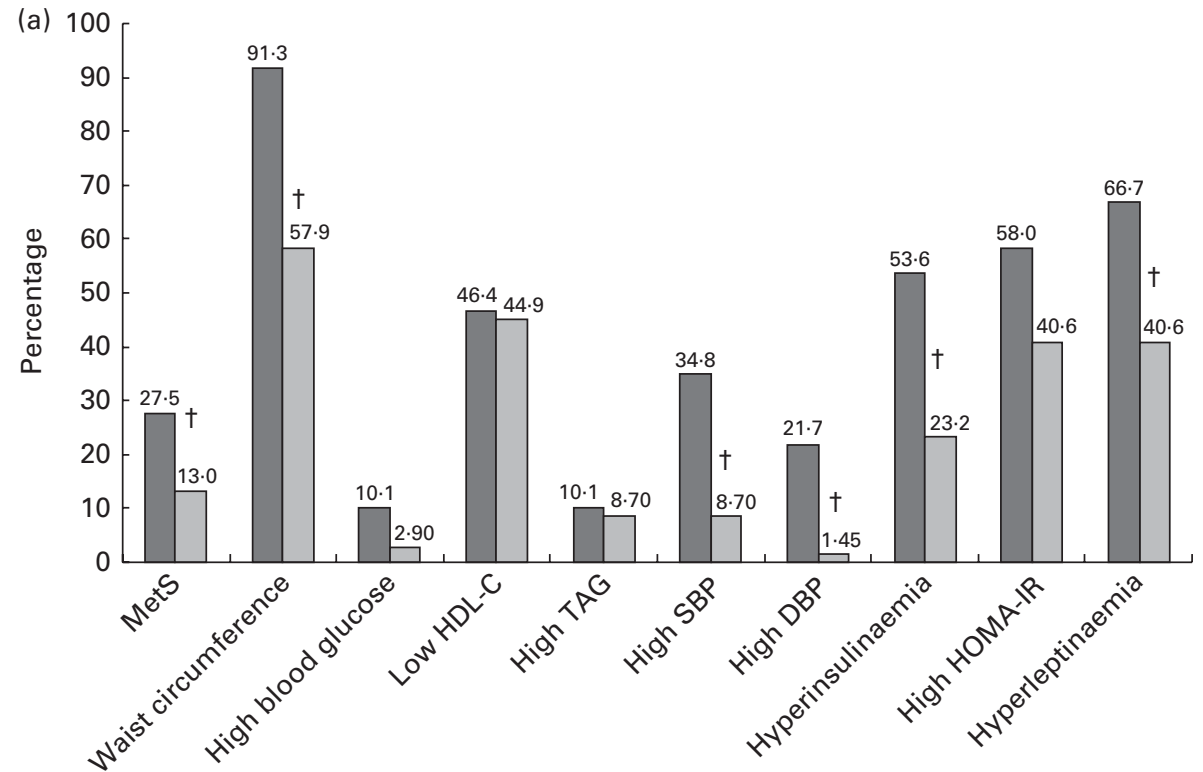

(b)

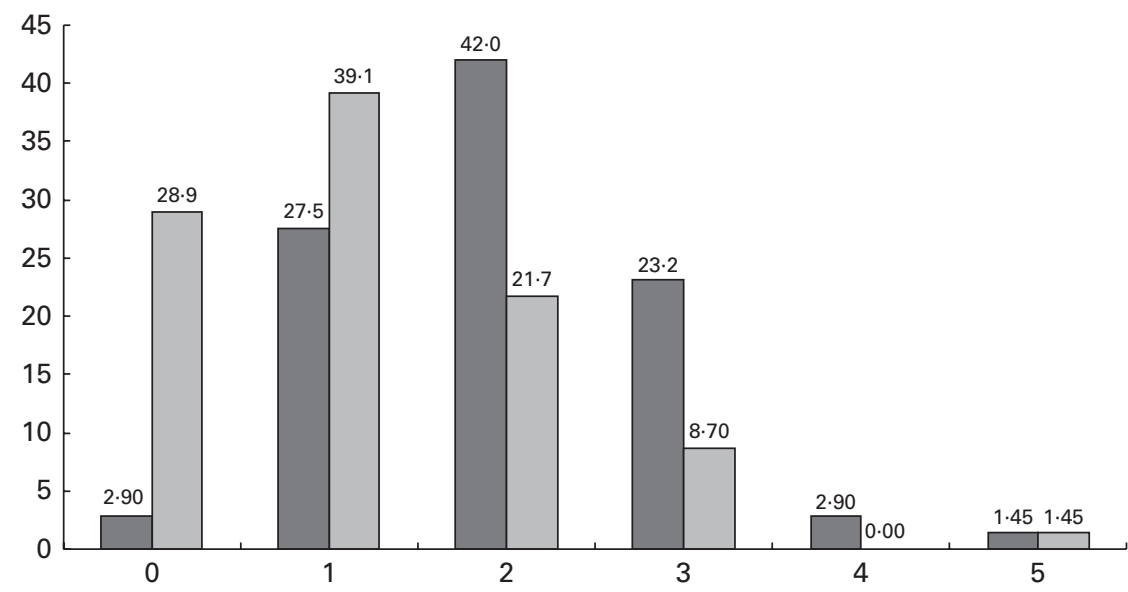

Fig. 1. The prevalence of metabolic syndrome (MetS) parameters, hyperinsulinaemia, high homeostasis model assessment for insulin resistance (HOMA-IR) and hyperleptinaemia (a) and the number of altered parameters in obese adolescents at baseline ( $\square$ ) and after therapy ( $\square$ ) (b). The prevalence altered parameters was obtained according to parameters established from International Diabetes Federation (IDF) criteria. $†$ Mean value was significantly different from that of baseline $\left(P<0.05\right.$; McNemar $\chi^{2}$ test). HDL-C, HDL-cholesterol; SBP, systolic blood pressure; DBP, diastolic blood pressure.

biomarkers of inflammation, leptin, the leptin:adiponectin ratio, PAI-1, cIMT and HOMA-AD were reduced. We also observed significant increases in adiponectin and the adiponectin:leptin ratio (Table 2).

\section{The effect of multicomponent therapy on the metabolic syndrome group}

After therapy, the MetS group presented significant reductions in anthropometric parameters and body composition (body mass, BMI, body fat, waist circumference, visceral and subcutaneous fat, and visceral:subcutaneous fat ratio; Table 1). Fat-free mass (\%) also increased in this group. Significant improvements in glucose, insulin, HOMA-IR and QUICKI were observed. With regard to the lipid profile, the MetS group showed reductions only in TAG and VLDL-C. Moreover,
SBP, DBP and MBP were significantly reduced. This group presented with significant decreases in leptin, PAI-1, hs-CRP, CIMT and HOMA-AD. Adiponectin also increased significantly in the MetS group (Table 2).

\section{Comparison between the groups}

At baseline, the MetS group presented significantly higher values for waist circumference, glucose, insulin, and SBP and MBP, but these differences did not remain after therapy (Table 1). However, this group also presented significantly higher VLDL-C and TAG levels and a lower HDL-C level at baseline and after therapy. After intervention, variations $(\Delta)$ in insulin, HOMA-IR, SBP, MBP, PAI-1 and HOMA-AD were significantly increased in the MetS group compared with the non-MetS group (Tables 1 and 2). 
Table 1. Anthropometric, metabolic and clinical data for the obese adolescents in the metabolic syndrome (MetS) and non-MetS groups during multicomponent therapy (Mean values and standard deviations; median values and minimum-maximum)

\begin{tabular}{|c|c|c|c|c|c|c|c|c|c|c|c|c|}
\hline & \multicolumn{6}{|c|}{ Non-MetS ( $n$ 50) } & \multicolumn{6}{|c|}{ MetS $(n 19)$} \\
\hline & \multicolumn{2}{|c|}{ Baseline } & \multicolumn{2}{|c|}{ After therapy } & \multicolumn{2}{|l|}{$\Delta$} & \multicolumn{2}{|c|}{ Baseline } & \multicolumn{2}{|c|}{ After therapy } & \multicolumn{2}{|l|}{$\Delta$} \\
\hline & Mean & SD & Mean & SD & Mean & SD & Mean & SD & Mean & SD & Mean & SD \\
\hline Age (years) & $17 \cdot 0$ & 1.43 & $17 \cdot 7$ & 1.43 & & & $16 \cdot 6$ & 1.59 & $17 \cdot 4$ & $1 \cdot 61$ & & \\
\hline Body mass (kg) & 102 & $15 \cdot 1$ & $91.5 \dagger$ & 14.9 & $-10 \cdot 2$ & 8.09 & 105 & $10 \cdot 9$ & $94 \cdot 1 \dagger$ & $13 \cdot 3$ & $-11 \cdot 3$ & $8 \cdot 19$ \\
\hline Height (m) & 1.67 & 0.08 & 1.67 & 0.08 & 0.00 & 0.01 & 1.66 & 0.07 & 1.66 & 0.07 & 0.00 & 0.01 \\
\hline BMI $\left(\mathrm{kg} / \mathrm{m}^{2}\right)$ & $36 \cdot 4$ & 5.05 & $32 \cdot 6 \dagger$ & 5.04 & $-3 \cdot 76$ & $2 \cdot 82$ & 38.6 & $5 \cdot 72$ & $34.4 \dagger$ & $6 \cdot 24$ & -4.26 & 3.03 \\
\hline Body fat (\%) & $43 \cdot 8$ & & $35.4 \dagger$ & & $-8 \cdot 23$ & & 45.4 & & $36.9 \dagger$ & & -8.51 & \\
\hline Fat-free mass (\%) & $56 \cdot 3$ & & $64.5 \dagger$ & & $8 \cdot 22$ & & $54 \cdot 6$ & & $63.1 \dagger$ & & 8.52 & \\
\hline Body fat $(\mathrm{kg})$ & 44.9 & $13 \cdot 3$ & $33 \cdot 1 \dagger$ & $12 \cdot 5$ & $-11 \cdot 8$ & 8.25 & $48 \cdot 3$ & $10 \cdot 9$ & $35.5 \dagger$ & $12 \cdot 5$ & $-12 \cdot 8$ & 8.88 \\
\hline Fat-free mass $(\mathrm{kg})$ & $56 \cdot 8$ & 9.91 & $58.6 \dagger$ & $9 \cdot 42$ & 1.55 & $5 \cdot 26$ & $57 \cdot 1$ & 4.99 & $59 \cdot 0$ & 5.90 & 1.93 & 5.33 \\
\hline Waist circumference $(\mathrm{cm})$ & 102 & 9.91 & $93.2 \dagger$ & $10 \cdot 9$ & $-8 \cdot 12$ & $9 \cdot 21$ & $108^{*}$ & 8.45 & $97 \cdot 0 \dagger$ & $12 \cdot 9$ & $-10 \cdot 9$ & 9.52 \\
\hline Visceral fat $(\mathrm{cm})$ & 4.42 & 1.50 & $2 \cdot 76 \dagger$ & 1.31 & -1.56 & 1.14 & 4.89 & 1.51 & $3.07 \dagger$ & 1.39 & -1.82 & 0.87 \\
\hline Subcutaneous fat $(\mathrm{cm})$ & 3.77 & 0.91 & $3 \cdot 12 \dagger$ & 0.98 & -0.69 & 0.74 & 3.78 & 0.80 & $3 \cdot 27 \dagger$ & 0.98 & -0.51 & 0.78 \\
\hline Visceral:subcutaneous fat & 1.23 & 0.49 & $0.96 \dagger$ & 0.49 & -0.22 & 0.50 & 1.35 & 0.50 & $0.98 \dagger$ & 0.43 & -0.37 & 0.25 \\
\hline Glucose (mmol/l) & 4.97 & 0.36 & 4.90 & 0.40 & -0.07 & 0.43 & $5 \cdot 17^{*}$ & 0.37 & $4.90 \dagger$ & 0.43 & -0.26 & 0.52 \\
\hline Insulin $(\mu \mathrm{U} / \mathrm{ml}) \ddagger$ & $15 \cdot 3$ & 5.66 & $12 \cdot 1 \dagger$ & $10 \cdot 2$ & $-3 \cdot 16$ & $10 \cdot 6$ & $20 \cdot 3^{*}$ & 11.4 & $13.4 \dagger$ & $11 \cdot 0$ & $-6 \cdot 87^{\star}$ & 8.41 \\
\hline \multicolumn{13}{|l|}{ HOMA-IR } \\
\hline Median & 3.24 & & $2 \cdot 27 \dagger$ & & -0.92 & & 3.89 & & $2 \cdot 09 \dagger$ & & $-1.84^{\star}$ & \\
\hline Minimum-maximum & $1 \cdot 24-6.59$ & & $0.73-22.1$ & & $-5 \cdot 01-18 \cdot 2$ & & $1.86-14.4$ & & $0.59-10.5$ & & $-4.64-5.08$ & \\
\hline QUICKI & 0.32 & 0.02 & $0.34 \dagger$ & 0.03 & 0.02 & 0.03 & 0.31 & $0 \cdot 19$ & $0.34 \dagger$ & 0.04 & 0.03 & 0.03 \\
\hline Total cholesterol (mmol/l) & $4 \cdot 01$ & 0.67 & $3 \cdot 81 \dagger$ & 0.71 & -0.20 & 0.48 & $4 \cdot 20$ & 0.67 & 3.94 & 0.85 & -0.25 & 0.55 \\
\hline $\mathrm{HDL}-\mathrm{C}(\mathrm{mmol} / \mathrm{l})$ & $1 \cdot 22$ & 0.26 & $1 \cdot 26$ & 0.30 & 0.04 & 0.16 & $1 \cdot 00^{*}$ & $0 \cdot 16$ & $1.03^{*}$ & 0.19 & 0.02 & 0.14 \\
\hline LDL-C (mmol/l) & $2 \cdot 34$ & 0.62 & $2 \cdot 18 \dagger$ & 0.58 & -0.17 & 0.39 & $2 \cdot 54$ & 0.58 & $2 \cdot 35$ & 0.59 & -0.19 & 0.49 \\
\hline VLDL-C (mmol/l) & 0.46 & 0.15 & $0.38 \dagger$ & 0.18 & -0.08 & 0.18 & $0.65^{*}$ & 0.32 & $0.57^{\star} \dagger$ & 0.31 & -0.08 & 0.15 \\
\hline TAG $(\mathrm{mmol} / \mathrm{l})$ & 0.99 & 0.34 & $0.82 \dagger$ & 0.39 & -0.17 & 0.39 & $1.42^{*}$ & 0.70 & $1 \cdot 23^{\star} \dagger$ & 0.68 & -0.19 & 0.32 \\
\hline \multicolumn{13}{|c|}{ Systolic blood pressure $(\mathrm{mmHg})$} \\
\hline Median & 120 & & $110 \dagger$ & & -5.00 & & $130^{\star}$ & & $120 \dagger$ & & $-20 \cdot 0^{\star}$ & \\
\hline Minimum-maximum & $100-190$ & & $100-130$ & & $-70 \cdot 0-20 \cdot 0$ & & $105-150$ & & $100-130$ & & $-40 \cdot 0-10 \cdot 0$ & \\
\hline \multicolumn{13}{|c|}{ Diastolic blood pressure $(\mathrm{mmHg})$} \\
\hline Median & $80 \cdot 0$ & & $70 \cdot 0 \dagger$ & & 0.00 & & $80 \cdot 0$ & & 80.0† & & -5.00 & \\
\hline Minimum-maximum & $70 \cdot 0-110$ & & $70 \cdot 0-90 \cdot 0$ & & $-40 \cdot 0-10 \cdot 0$ & & $70 \cdot 0-100$ & & $70 \cdot 0-80 \cdot 0$ & & $-20 \cdot 0-10 \cdot 0$ & \\
\hline \multicolumn{13}{|c|}{ Median blood pressure $(\mathrm{mmHg})$} \\
\hline Median & 93.3 & & $86.7 \dagger$ & & -3.33 & & $96 \cdot 7^{\star}$ & & $93.3 \dagger$ & & $-6 \cdot 67^{\star}$ & \\
\hline Minimum-maximum & $80 \cdot 0-136$ & & $80-103$ & & $-50 \cdot 0-13 \cdot 3$ & & $83 \cdot 3-113$ & & $80 \cdot 0-96 \cdot 7$ & & $-20 \cdot 0-10 \cdot 0$ & \\
\hline \multicolumn{13}{|l|}{ Total energy intake } \\
\hline kcal & 2070 & 819 & $1387 \dagger$ & 412 & -666 & 882 & 1813 & 374 & 1353 & 313 & -336 & 649 \\
\hline kJ & 8661 & 3427 & 5803 & 1724 & -2786 & 3690 & 7585 & 1565 & 5661 & 1309 & -1406 & 2715 \\
\hline
\end{tabular}

HOMA-IR, homeostasis model assessment for insulin resistance; QUICKI, quantitative insulin sensitivity check index; HDL-C, HDL-cholesterol, LDL-C, LDL-cholesterol; VLDL-C, VLDL-cholesterol. * Mean value was significantly different from that of non-MetS group at the same time point $(P<0.05)$.

† Mean value was significantly different from that of baseline $(P<0.05)$.

‡To convert insulin in $\mu \mathrm{U} / \mathrm{ml}$ to pmol/l, multiply by 6.945 . 


\section{Correlations and regression analysis}

Entire group. At baseline, the number of MetS parameters was correlated with body mass, BMI, waist circumference and HOMA-IR (Table 3). The leptin concentration and leptin: adiponectin ratio and adiponectin:leptin ratio were correlated with BMI and body fat ( $\%$ and $\mathrm{kg}$ ). PAI- 1 was correlated with SBP, DBP and MBP. hs-CRP was correlated with body mass, BMI, body fat (\% and $\mathrm{kg}$ ) and waist circumference.

A positive correlation of the number of metabolic alterations with leptin and the leptin:adiponectin ratio and a negative correlation with the adiponectin:leptin ratio was also observed at the end of the therapy. In the regression analysis present in Table 4, leptin and the adiponectin:leptin ratio revealed a potential association with the number of MetS parameters in obese adolescents at the end of the therapy.

The $\Delta$ in leptin correlated with the $\Delta$ in body mass, BMI and body fat ( $\mathrm{kg}$ and \%) (Table 3 ). The $\Delta$ in adiponectin correlated with the $\Delta$ in glucose, HOMA-IR and TAG. The $\Delta$ in the leptin:adiponectin ratio correlated with the $\Delta$ in body fat (\% and $\mathrm{kg}$ ) and HOMA-AD, and the $\Delta$ in the adiponectin:leptin ratio correlated with the $\Delta$ in body mass, BMI and body fat ( $\mathrm{kg}$ and \%).

Non-metabolic syndrome group. At baseline, the number of MetS parameters was correlated with body mass, BMI, body fat, waist circumference and PAI-1 (Table 3). After weight loss intervention, the $\Delta$ in leptin was correlated with the $\Delta$ in body mass, BMI and body fat, and the $\Delta$ in the leptin: adiponectin ratio was correlated with body fat.

Metabolic syndrome group. At baseline, the number of MetS parameters was correlated with glucose, total cholesterol, LDL-C and cIMT (Table 3). cIMT was correlated with total cholesterol and LDL-C (Table 3). After weight loss intervention, the $\Delta$ in the adiponectin:leptin ratio was correlated with the $\Delta$ in glucose, QUICKI, total cholesterol, LDL-C and SBP. We also observed that the $\Delta$ in CIMT was correlated with the $\Delta$ in QUICKI, total cholesterol and LDL-C.

\section{Discussion}

The first aim of the present study was to verify whether multicomponent therapy effectively improved the MetS parameters, inflammation and cardiovascular risks in obese adolescents. At baseline, the obese individuals with the MetS had significantly higher waist circumference, glucose, insulin, SBP and MBP, VLDL-C, and TAG and lower HDL-C compared with obese individuals without the MetS. The most important finding was that this therapy promoted significant reductions in the prevalence of the MetS, the cardiometabolic profile, inflammation, pro-thrombotic factor and cIMT in obese adolescents after 1 year of intervention. All of these improvements are important in controlling obesity, CVD and subclinical atherosclerotic processes, because the presence of this cluster of cardiometabolic abnormalities during childhood raises the risk of CVD during adulthood ${ }^{(39)}$.

The MetS diagnoses in paediatric patients have been associated with a 2 -fold increase in the risk of developing high cIMT during adulthood ${ }^{(39)}$. cIMT has been considered 
Table 3. Correlations between variables

\begin{tabular}{|c|c|c|c|c|c|}
\hline & $r$ & $P$ & & $r$ & $P$ \\
\hline \multicolumn{3}{|l|}{ Entire group (baseline) } & \multicolumn{3}{|l|}{ Entire group (after therapy) } \\
\hline Number of MetS parameters & & & Number of MetS parameters & & \\
\hline Body mass $(\mathrm{kg})$ & 0.35 & $<0.05$ & Leptin (ng/ml) & 0.47 & $<0.05$ \\
\hline BMI $\left(\mathrm{kg} / \mathrm{m}^{2}\right)$ & 0.34 & $<0.05$ & Leptin:adiponectin ratio & 0.44 & $<0.05$ \\
\hline Waist circumference $(\mathrm{cm})$ & 0.44 & $<0.05$ & Adiponectin:leptin ratio & -0.39 & $<0.05$ \\
\hline HOMA-IR & 0.30 & $<0.05$ & $\Delta$ Leptin $(\mathrm{ng} / \mathrm{ml})$ & & \\
\hline Leptin (ng/ml) & & & $\Delta$ Body mass $(\mathrm{kg})$ & 0.34 & $<0.05$ \\
\hline BMl $\left(\mathrm{kg} / \mathrm{m}^{2}\right)$ & 0.39 & $<0.05$ & $\Delta \mathrm{BMI}\left(\mathrm{kg} / \mathrm{m}^{2}\right)$ & 0.36 & $<0.05$ \\
\hline Body fat (\%) & 0.51 & $<0.05$ & $\Delta$ Body fat $(\mathrm{kg})$ & 0.42 & $<0.05$ \\
\hline Body fat $(\mathrm{kg})$ & 0.43 & $<0.05$ & $\Delta$ Body fat $(\%)$ & 0.39 & $<0.05$ \\
\hline Leptin:adiponectin ratio & & & $\Delta$ Adiponectin $(\mu \mathrm{g} / \mathrm{l})$ & & \\
\hline BMI $\left(\mathrm{kg} / \mathrm{m}^{2}\right)$ & 0.40 & $<0.05$ & $\Delta$ Glucose $(\mathrm{mmol} / \mathrm{l})$ & -0.36 & $<0.05$ \\
\hline Body fat (\%) & 0.48 & $<0.05$ & $\Delta$ HOMA-IR & -0.32 & $<0.05$ \\
\hline Body fat (kg) & 0.43 & $<0.05$ & $\Delta \mathrm{TAG}(\mathrm{mmol} / \mathrm{l})$ & -0.32 & $<0.05$ \\
\hline Adiponectin:leptin ratio & & & $\Delta$ Leptin:adiponectin ratio & & \\
\hline BMI $\left(\mathrm{kg} / \mathrm{m}^{2}\right)$ & -0.36 & $<0.05$ & $\Delta$ Body fat (\%) & 0.38 & $<0.05$ \\
\hline Body fat (\%) & -0.47 & $<0.05$ & $\Delta$ Body fat $(\mathrm{kg})$ & 0.37 & $<0.05$ \\
\hline Body fat (kg) & -0.41 & $<0.05$ & $\Delta$ HOMA-AD & 0.35 & $<0.05$ \\
\hline PAl-1 (ng/ml) & & & $\Delta$ Adiponectin:leptin ratio & & \\
\hline SBP (mmHg) & 0.46 & $<0.05$ & $\Delta$ Body mass $(\mathrm{kg})$ & -0.33 & $<0.05$ \\
\hline $\mathrm{DBP}(\mathrm{mmHg})$ & 0.45 & $<0.05$ & $\Delta \mathrm{BMI}\left(\mathrm{kg} / \mathrm{m}^{2}\right)$ & -0.32 & $<0.05$ \\
\hline MBP (mmHg) & 0.50 & $<0.05$ & $\Delta$ Body fat $(\mathrm{kg})$ & -0.31 & $<0.05$ \\
\hline hs-CRP (ng/ml) & & & $\Delta$ Body fat $(\%)$ & -0.30 & $<0.05$ \\
\hline Body mass $(\mathrm{kg})$ & 0.43 & $<0.05$ & Non-MetS (after therapy) & & \\
\hline BMI $\left(\mathrm{kg} / \mathrm{m}^{2}\right)$ & 0.57 & $<0.05$ & $\Delta$ Leptin(ng/ml) & & \\
\hline Body fat (\%) & 0.49 & $<0.05$ & $\Delta$ Body mass $(\mathrm{kg})$ & 0.35 & $<0.05$ \\
\hline Body fat $(\mathrm{kg})$ & 0.53 & $<0.05$ & $\Delta \mathrm{BMI}\left(\mathrm{kg} / \mathrm{m}^{2}\right)$ & 0.39 & $<0.05$ \\
\hline Waist circumference $(\mathrm{cm})$ & 0.35 & $<0.05$ & $\Delta$ Body fat $(\%)$ & 0.39 & $<0.05$ \\
\hline Non-MetS (baseline) & & & $\Delta$ Body fat $(\mathrm{kg})$ & 0.42 & $<0.05$ \\
\hline Number of MetS parameters & & & $\Delta$ Leptin:adiponectin ratio & & \\
\hline Body mass $(\mathrm{kg})$ & 0.46 & $<0.05$ & $\Delta$ Body fat $(\%)$ & 0.47 & $<0.05$ \\
\hline BMI $\left(\mathrm{kg} / \mathrm{m}^{2}\right)$ & 0.43 & $<0.05$ & $\Delta$ Body fat $(\mathrm{kg})$ & 0.39 & $<0.05$ \\
\hline Body fat (kg) & 0.35 & $<0.05$ & MetS (after therapy) & & \\
\hline Waist circumference $(\mathrm{cm})$ & 0.45 & $<0.05$ & $\Delta$ Adiponectin:leptin ratio & & \\
\hline PAl-1 (ng/ml) & 0.43 & $<0.05$ & $\Delta$ Glucose $(\mathrm{mmol} / \mathrm{l})$ & -0.52 & $<0.05$ \\
\hline MetS (baseline) & & & $\Delta$ QUICKI & 0.51 & $<0.05$ \\
\hline Number of MetS parameters & & & $\Delta$ Total cholesterol $(\mathrm{mmol} / \mathrm{l})$ & -0.57 & $<0.05$ \\
\hline Glucose (mmol/l) & 0.49 & $<0.05$ & $\Delta$ LDL-C $(\mathrm{mmol} / \mathrm{l})$ & -0.59 & $<0.05$ \\
\hline Total cholesterol $(\mathrm{mmol} / \mathrm{l})$ & 0.63 & $<0.05$ & $\Delta \mathrm{SBP}(\mathrm{mmHg})$ & -0.47 & $<0.05$ \\
\hline LDL-C (mmol/l) & 0.63 & $<0.05$ & $\Delta \mathrm{clMT}(\mathrm{mm})$ & & \\
\hline clMT $(\mathrm{mm})$ & 0.55 & $<0.05$ & $\Delta$ QUICKI & -0.56 & $<0.05$ \\
\hline clMT (mm) & & & $\Delta$ Total cholesterol $(\mathrm{mmol} / \mathrm{l})$ & 0.55 & $<0.05$ \\
\hline Total cholesterol (mmol/l) & 0.56 & $<0.05$ & $\Delta \mathrm{LDL}-\mathrm{C}(\mathrm{mmol} / \mathrm{l})$ & 0.61 & $<0.05$ \\
\hline LDL-C (mmol/l) & 0.54 & $<0.05$ & & & \\
\hline
\end{tabular}

HOMA-IR, homeostasis model assessment for insulin resistance; HOMA-AD, homeostasis model assessment-adiponectin; PAI-1, plasminogen activator inhibitor 1; SBP, systolic blood pressure; DBP, diastolic blood pressure; MBP, mean blood pressure; hs-CRP, high-sensitivity C-reactive protein; MetS, metabolic syndrome; QUICKI, quantitative insulin sensitivity check index; LDL-C, LDL-cholesterol; cIMT, carotid intima-media thickness.

to be an important early screening tool for assessing subclinical manifestations of CVD, because it is the first subclinical sign of atherosclerosis and results from endothelial dysfunction that creates lipid deposits in the intima of systemic arteries ${ }^{(40)}$. In the present study, cIMT was reduced in both groups (MetS and non-MetS). It is important to note that the number of MetS parameters was correlated with cIMT in MetS obese adolescents. Our findings are consistent with those of other studies in which adults with the MetS presented higher cIMT values compared with patients without the $\mathrm{MetS}^{(41)}$. Leite et $a l .{ }^{(42)}$ demonstrated that adolescents with the MetS have increased cIMT compared with normalweight and overweight adolescents. These results reinforce the link between obesity, cardiometabolic abnormalities and
CVD, and it is crucial that they are considered during childhood in clinical practice.

Importantly, obese adolescents with the MetS did not show reductions in total cholesterol and LDL-C after therapy; such improvements were observed only in the non-MetS group (Table 1). The present findings also demonstrate that total cholesterol and LDL-C were correlated with higher cIMT values in the MetS patients (Table 3). These findings suggest that in obese adolescents with the MetS, the clustering of metabolic abnormalities and central obesity, two key factors related to the physiopathology of the MetS, may have a resistance effect on lipid metabolism in contrast with adolescents without the MetS, rendering metabolic control more difficult $^{(43)}$. 
Table 4. Simple regression analysis of leptin and the adiponectin:leptin ratio and the number of metabolic alterations in the obese adolescents (Beta values and confidence limits)

\begin{tabular}{|c|c|c|c|c|}
\hline & $\beta$ & $P$ & $\begin{array}{c}-95.0 \% \\
\text { Confidence } \\
\text { limit }\end{array}$ & $\begin{array}{c}+95.0 \% \\
\text { Confidence } \\
\text { limit }\end{array}$ \\
\hline Leptin (ng/ml) & 0.31 & 0.01 & 0.08 & 0.56 \\
\hline Adiponectin:leptin ratio & -0.37 & 0.00 & -0.63 & -0.14 \\
\hline
\end{tabular}

Furthermore, we observed significant improvements in adiponectin, leptin, PAI-1, hs-CRP, HOMA-AD and HOMA-IR for both groups after therapy (Tables 1 and 2), contributing to the control of the pro-inflammatory state. Conversely, only the non-MetS adolescents presented significant improvements in adiponectin:leptin ratio and leptin:adiponectin ratio (Table 2). The modulation of the adiponectin:leptin ratio is an important factor for consideration in MetS patients, because this biomarker was found to be significantly correlated with the MetS parameters in the present study, especially the atherogenic lipid profile, which did not improve after intervention in the MetS group (Tables 2 and 3). Moreover, it has recently been demonstrated that an increase in the adiponectin:leptin ratio is an independent predictor of cIMT reduction in obese adolescents after weight $\operatorname{loss}^{(9)}$. Together, these data demonstrate the key role of the adiponectin:leptin ratio in modulating cardiometabolic parameters and CVD progression in obese adolescents.

Previous findings have demonstrated that the leptin concentration is positively associated with the MetS parameters in children, adolescents and adults ${ }^{(44-46)}$. Thus, hyperleptinaemia may be associated with CVD, endothelial dysfunction and myocardial infarction ${ }^{(13,47)}$. Importantly, in the present analysis, the prevalence of hyperleptinaemia was significantly reduced after therapy (Fig. 1(a)).

However, adiponectin is an anti-inflammatory adipokine that plays an important role in metabolic and cardiovascular homeostases. This adipokine exerts anti-atherosclerotic effects via the inhibition of pro-inflammatory cytokines, such as TNF- $\alpha$ and the stimulation of anti-inflammatory cytokines. Moreover, adiponectin is an insulin sensitiser that contributes to the delay and/or prevention of insulin resistance and type 2 diabetes ${ }^{(43)}$. The modulation of both the leptin and adiponectin concentrations is an important strategy for controlling the MetS.

For the second aim of the present study, we explored the possible relationships between pro/anti-inflammatory adipokines, the MetS parameters and cIMT in obese adolescents. Among the inflammatory markers, leptin and leptin:adiponectin ratio, and adiponectin:leptin ratio were correlated with the number of MetS parameters at the end of the therapy. However, in the regression analysis, only leptin and the adiponectin:leptin ratio showed potential associations with the number of MetS parameters in obese adolescents.

Importantly, the number of MetS parameters was positively correlated with increases in waist circumference, body mass, BMI and HOMA-IR for the entire group, confirming the role of adipose tissue and insulin resistance in promoting cardiometabolic alterations ${ }^{(11)}$. Thus, clinical strategies for promoting weight loss and fat reduction can directly influence and control the MetS parameters, as was observed in the present study.

Another important finding is that changes in cIMT were correlated with changes in total cholesterol, LDL-C and QUICKI in the MetS group after therapy. The results of a previous longitudinal study showing that LDL-C is a predictor of CIMT progression and demonstrating that cIMT progression increases by $3 \%$ for each $1 \mathrm{mg} / \mathrm{dl}(0.0259 \mathrm{mmol} / \mathrm{l})$ increase in LDL-C are in agreement with our findings. Additionally, the authors also found a higher incidence of cIMT progression in adolescents with higher levels of LDL-C and total cholesterol ${ }^{(48)}$.

Correlations between the adiponectin:leptin ratio and the MetS parameters, such as glucose, QUICKI, total cholesterol, LDL-C and SBP, after therapy in the MetS group were validated in the present study. To confirm this hypothesis, we verified the role of adiponectin in controlling glucose metabolism, because it was found to be negatively correlated with glucose and HOMA-IR after the weight loss intervention for the entire group. These data reinforce the influence of the adiponectin: leptin ratio on the MetS parameters and cardiovascular risk in the MetS patients, as has been previously reported in other studies $^{(49,50)}$.

Additionally, in the present analysis, we verified that the baseline and $\Delta$ in leptin and the adiponectin:leptin ratio and leptin:adiponectin ratio were correlated with body mass, BMI and body fat, demonstrating the effects of both weight control and fat loss on the attenuation of the inflammatory process. Moreover, changes in the leptin:adiponectin ratio were correlated with the changes in insulin resistance estimated by the HOMA-AD. Accordingly, the results of the HELENA study that evaluated 1053 European adolescents indicated that leptin is a risk factor for insulin resistance in both boys and girls ${ }^{(46)}$.

It is also important to note that in the present analysis, a correlation was found between PAI-1 and blood pressure in the entire group as well as between PAI-1 and the number of MetS parameters in the non-MetS group. In agreement with these findings, a positive relationship between PAI-1 and the MetS parameters has previously been demonstrated, including the HOMA-IR and blood pressure, in obese adolescents ${ }^{(51)}$. PAI-1 is thought to be a pro-thrombotic adipokine involved in thrombus formation and cardiovascular risk and an important biomarker for vascular endothelial function, and it is increased in obese adolescents diagnosed with the MetS because of the inflammatory state caused by the excessive adipose tissue ${ }^{(51,52)}$.

Some limitations must be considered in the interpretation of these findings. A large sample size is needed to confirm the results observed in the present study. Additionally, further investigations should be performed of other inflammatory parameters. Nonetheless, the strengths of the present study include the positive effects of multicomponent therapy, such as nutrition, physical exercise, psychological and clinical support, that were observed, indicating the effectiveness of this clinical strategy for controlling the MetS, inflammation and cardiovascular risks in obese adolescents. 
In conclusion, the multicomponent therapy promoted significant improvements in the metabolic parameters, the inflammatory profile and cIMT. However, the MetS patients showed resistance in the attenuation of the atherogenic lipid profile and leptin:adiponectin ratio and adiponectin:leptin ratio. These results suggest that the MetS patients have increased cardiovascular risks and that it is important to attempt to control the inflammatory process associated with obesity in clinical practice to improve adolescent health. Additionally, we were able to show the relationship of the MetS parameters with inflammatory markers and CIMT in obese adolescents.

CNPq (no. 141533/2012-9), CAPES (no. AUX-PE-PNPD 2566/2011), FAPESP (no. 2011/50 356-0; 2011/50 414-0; 2013/ 041364), UNIFESP, AFIP, CEPE and CEMSA supported the Interdisciplinary Obesity Program. Special thanks go to the volunteers and their families.

\section{Acknowledgements}

The authors' contributions are as follows: D. C. L. M., A. d. P., R. M. S. C., P. L. S. and A. R. D. designed the experiment, collected and analysed the data, and wrote the manuscript; J. C., B. D. M. N., J. P. C.-F., F. C. C., L. M. O. and C. M. O. N. designed the experiment, and collected and analysed the data; M. T. d. M., S. T. and A. R. D. conceived the study, participated in its design and coordination, and helped to draft the manuscript. All authors approved the final version of the study

The authors declare that they have no conflicts of interest.

\section{References}

1. Cali AMG \& Caprio S (2008) Obesity in children and adolescents. J Clin Endocrinol Metab 93, S31-S36.

2. Alexopoulos N, Katritsis D \& Raggi P (2014) Visceral adipose tissue as a source of inflammation and promoter of atherosclerosis. Atherosclerosis 233, 104-112.

3. Garg PK, Biggs ML, Carnethon M, et al. (2014) Metabolic syndrome and risk of incident peripheral artery disease: the cardiovascular health study. Hypertension 63, 413-419.

4. Corgosinho FC, de Piano A, Sanches PL, et al. (2012) The role of PAI-1 and adiponectin on the inflammatory state and energy balance in obese adolescents with metabolic syndrome. Inflammation 35, 944-951.

5. Gami AS, Witt BJ, Howard DE, et al. (2007) Metabolic syndrome and risk of incident cardiovascular events and death: a systematic review and meta-analysis of longitudinal studies. I Am Coll Cardiol 49, 403-414.

6. Santaliestra-Pasías AM, Mouratidou T, Huybrechts I, et al. (2014) Increased sedentary behaviour is associated with unhealthy dietary patterns in European adolescents participating in the HELENA study. Eur J Clin Nutr 68, 300-308.

7. Jiménez-Pavón D, Sesé MA, Huybrechts I, et al. (2013) Dietary and lifestyle quality indices with/without physical activity and markers of insulin resistance in European adolescents: the HELENA study. Br J Nutr 110, 1919-1925.

8. Sanches PL, de Mello MT, Elias N, et al. (2014) Hyperleptinemia: implications on the inflammatory state and vascular protection in obese adolescents submitted to an interdisciplinary therapy. Inflammation 37, 35-43.
9. Masquio DC, de Piano A, Sanches PL, et al. (2013) The effect of weight loss magnitude on pro-/anti-inflammatory adipokines and carotid intima-media thickness in obese adolescents engaged in interdisciplinary weight loss therapy. Clin Endocrinol (Oxf) 79, 55-64.

10. Dâmaso AR, de Piano A, Campos RM, et al. (2013) Multidisciplinary approach to the treatment of obese adolescents: effects on cardiovascular risk factors, inflammatory profile, and neuroendocrine regulation of energy balance. Int $J$ Endocrinol 2013, 541032.

11. González M, del Mar Bibiloni M, Pons A, et al. (2012) Inflammatory markers and metabolic syndrome among adolescents. Eur J Clin Nutr 66, 1141-1145.

12. Zalesin KC, Franklin BA, Miller WM, et al. (2011) Impact of obesity on cardiovascular disease. Med Clin North Am $\mathbf{9 5}$, 919-937.

13. Chai SB, Sun F, Nie XL, et al. (2014) Leptin and coronary heart disease: a systematic review and meta-analysis. Atherosclerosis 233, 3-10.

14. Pérez CM, Ortiz AP, Fuentes-Mattei E, et al. (2014) High prevalence of cardiometabolic risk factors in Hispanic adolescents: correlations with adipocytokines and markers of inflammation. J Immigr Minor Health 16, 865-873.

15. Gidding SS, Palermo RA, DeLoach S, et al. (2014) Associations of cardiac structure with obesity, blood pressure, inflammation, and insulin resistance in African-American adolescents. Pediatr Cardiol 35, 307-314.

16. Tanner JM \& Whitehouse RH (1976) Clinical longitudinal standards for height, weight, weight velocity and stages of puberty. Arch Dis Child 51, 170-179.

17. Centers for Disease Control and Prevention (CDC) (2002) Prevalence of Overweight Among Children and Adolescents: United States 1999-2002. Hyattsville: National Center for Health Statistics. http://www.cdc.gov/nchs/products/pubs/ pubd/hestats/overwght99.htm (accessed 29 January 2013).

18. Zimmet P, Alberti KG, Kaufman F, et al. (2007) The metabolic syndrome in children and adolescents - an IDF consensus report. Pediatr Diabetes $\mathbf{8}, 299-306$.

19. Lohman TG, Roche AF \& Martorrel R (1991) Anthropometric Standardization Reference Manual. Illinois: Human Kinetic Books.

20. Fields DA, Higgins PB \& Radley D (2005) Air-displacement plethysmography: here to stay. Curr Opin Clin Nutr Metab Care 8, 624-629.

21. Ribeiro-Filho FF, Faria AN, Azjen S, et al. (2003) Methods of estimation of visceral fat: advantages of ultrasonography. Obes Res 11, 1488-1494.

22. Guardamagna O, Abello F, Saracco P, et al. (2009) Endothelial activation, inflammation and premature atherosclerosis in children with familial dyslipidemia. Atherosclerosis 207, 471-475.

23. Gutin B, Ramsey L, Barbeau P, et al. (1999) Plasma leptin concentrations in obese children: changes during 4-mo periods with and without physical training. Am J Clin Nutr 69, 388-394.

24. Matthews DR, Hosker JP, Rudenski AS, et al. (1985) Homeostasis model assessment: insulin resistance and $\beta$-cell function from fasting plasma glucose and insulin concentrations in man. Diabetologia 28, 412-419.

25. Matsuhisa M, Yamasaki Y, Emoto M, et al. (2007) A novel index of insulin resistance determined from the homeostasis model assessment index and adiponectin levels in Japanese subjects. Diabetes Res Clin Pract 77, 151-154.

26. Katz A, Nambi SS, Mather K, et al. (2000) Quantitative insulin sensitivity check index: a simple, accurate method 
for assessing insulin sensitivity in humans. J Clin Endocrinol Metab 85, 2402-2410.

27. Keskin M, Kurtoglu S, Kendirci M, et al. (2005) Homeostasis model assessment is more reliable than the fasting glucose/ insulin ratio and quantitative insulin sensitivity check index for assessing insulin resistance among obese children and adolescents. Pediatrics 115, e500-e503.

28. Brazilian Society of Pediatrics (2012) Obesity in Childhood and Adolescence - Guidance Manual. São Paulo: Scientific Department of Nutrition.

29. Philippi ST, Latterza AR, Cruz ATR, et al. (1999) Adapted food pyramid: guide to food choices. Braz J Nutr 12, 65-80.

30. NRC (2001) Dietary Reference Intake: Applications in Dietary Assessment. Washington, DC: National Academic Press.

31. Donnelly JE, Blair SN, Jakicic JM, et al. (2009) Appropriate physical activity intervention strategies for weight loss and prevention of weight regain for adults. Med Sci Sports Exerc 41, 459-471.

32. Foschini D, Araújo RC, Bacurau RF, et al. (2010) Treatment of obese adolescents: the influence of periodization models and ACE genotype. Obesity 18, 766-772.

33. Dâmaso AR, da Silveira Campos RM, Caranti DA, et al. (2014) Aerobic plus resistance training was more effective in improving the visceral adiposity, metabolic profile and inflammatory markers than aerobic training in obese adolescents. J Sports Sci 32, 1435-1445.

34. Gorenstein C \& Andrade L (1996) Validation of a Portuguese version of the beck depression inventory and the state-trait anxiety inventory in Brazilian subjects. Braz J Med Biol 29, 453-457.

35. Spielberger CD, Gorsuch RL \& Lushene E (1970) Manual for the State-Trait Anxiety Inventory ("Self-Evaluation Questionnaire"). Palo Alto, CA: Consulting Psychologist Press.

36. Cooper PJ, Taylor MJ, Cooper Z, et al. (1987) The development and validation of the body shape questionnaire. Int $J$ Eat Disord 6, 485-494.

37. Freitas S, Lopes C, Coutinho W, et al. (2001) Translation and adaptation to Portuguese of the Binge Eating Scale. BrazJ Psych 23, $215-220$.

38. Cordás TA \& Hochgraf PB (1993) BITE: instrument for the assessment of bulimia nervosa - Version into Portuguese. Braz J Psych 42, 141-144.

39. Magnussen CG, Koskinen J, Chen W, et al. (2010) Pediatric metabolic syndrome predicts adulthood metabolic syndrome, subclinical atherosclerosis, and type 2 diabetes mellitus but is no better than body mass index alone: the Bogalusa Heart Study and the Cardiovascular Risk in Young Finns Study. Circulation 122, 1604-1611.
40. de Lima Sanches P, de Mello MT, Elias N, et al. (2011) Improvement in HOMA-IR is an independent predictor of reduced carotid intima-media thickness in obese adolescents participating in an interdisciplinary weight-loss program. Hypertens Res 34, 232-238.

41. Aydin M, Bulur S, Alemdar R, et al. (2013) The impact of metabolic syndrome on carotid intima media thickness. Eur Rev Med Pharmacol Sci 17, 2295-2301.

42. Leite A, Santos A, Monteiro M, et al. (2012) Impact of overweight and obesity in carotid intima-media thickness of Portuguese adolescents. Acta Paediatr 101, e115-e121.

43. Fuentes E, Fuentes F, Vilahur G, et al. (2013) Mechanisms of chronic state of inflammation as mediators that link obese adipose tissue and metabolic syndrome. Mediators Inflamm 2013, 136584.

44. Smits MM, Woudstra P, Utzschneider KM, et al. (2013) Adipocytokines as features of the metabolic syndrome determined using confirmatory factor analysis. Ann Epidemiol 23, 415-421.

45. Wang Q, Yin J, Xu L, et al. (2013) Prevalence of metabolic syndrome in a cohort of Chinese schoolchildren: comparison of two definitions and assessment of adipokines as components by factor analysis. BMC Public Health 13, 249.

46. Jiménez-Pavón D, Sesé MA, Valtueña J, et al. (2014) Leptin, vitamin D, and cardiorespiratory fitness as risk factors for insulin resistance in European adolescents: gender differences in the HELENA study. Appl Physiol Nutr Metab 39, $530-537$.

47. Nakamura K, Fuster JJ \& Walsh K (2013) Adipokines: a link between obesity and cardiovascular disease. J Cardiol 63 , $250-259$.

48. Toledo-Corral CM, Davis JN, Alderete TL, et al. (2011) Subclinical atherosclerosis in Latino youth: progression of carotid intima-media thickness and its relationship to cardiometabolic risk factors. J Pediatr 158, 935-940.

49. Mirza S, Qu HQ, Li Q, et al. (2011) Adiponectin/leptin ratio and metabolic syndrome in a Mexican American population. Clin Invest Med 34, E290.

50. Vega GL \& Grundy M (2013) Metabolic risk susceptibility in men is partially related to adiponectin/leptin ratio. $J$ Obes 2013, 409679.

51. Masquio DC, de Piano A, Campos RM, et al. (2014) Saturated fatty acid intake can influence increase in plasminogen activator inhibitor-1 in obese adolescents. Horm Metab Res $\mathbf{4 6}$, $245-251$.

52. Wei Y, Liu G, Yang J, et al. (2013) The association between metabolic syndrome and vascular endothelial dysfunction in adolescents. Exp Ther Med 5, 1663-1666. 